\title{
Debate: The Biology of Breast Cancer in Young Women Is Unique
}

Section Editors' Note: This article is based on a debate that took place during the European School of Oncology (ESO) Inside Track Conference "Breast Cancer in Young Women (BCY1)" which took place from 8-10 ${ }^{\text {th }}$ November 2012 in Dublin, Ireland.

Breast cancer in young women frequently presents with an aggressive phenotype, leading to a poorer prognosis than in older women. The critical issue centres on whether the drivers of this "poor-prognosis" phenotype in young women represent a distinct biology or reflect an overrepresentation of molecular and cellular processes that underpin aggressive disease in all women with this common malignancy. Addressing whether or not the biology of breast cancer in young women is truly unique is an important question, as it increases our understanding of the disease process, while informing the provision of appropriate optimal quality care for the young breast cancer patient. The Oncologist 2013;18:e13-e15

\section{IN FAVouR}

\section{By Marco Colleoni}

Division of Medical Senology, European Institute of Oncology, Milan, Italy

Breast cancer at a young age has been reported to pursue a more aggressive clinical course and to be associated with a poorer prognosis compared with disease in older women [1]. Factors influencing poor

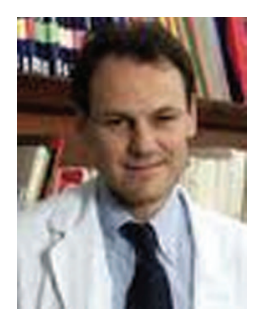

Marco Colleoni prognosis in this patient group include higher tumour grade at diagnosis, high tumour proliferation, pronounced vessel-invading disease, increased expression of HER-2 (ERB-B2) and reduced expression of both estrogen (ER) and progesterone receptor (PR) [2].

Both immunohistochemical (IHC) and molecular classifications have been employed to address whether cancer biology defines a unique disease in young women with breast cancer [3-6]. Previous research has identified four subtypes: luminal A (lessaggressive subtype), luminal B, HER-2 enriched and triple negative (more-aggressive subtypes), which have prognostic relevance $[6,7]$. Evaluation of these four subtypes in a cohort of 2,970 young patients, which included a subset of "very young women" ( $<35$ years) with breast cancer, indicated that there were significantly more patients with triple-negative subtypes and significantly fewer luminal A subtypes in the "very young" cohort when compared with the "less young" women [8]. Other studies have also identified luminal subtypes in older patients [9], with triple-negative subtypes overrepresented in women younger than 40 years of age [10]. The finding that "very young" patients with tumours classified as luminal B, HER-2 enriched and triple-negative subtypes were at increased risk of relapse when compared with older patients with the same subtype [8], suggests that younger patients with breast cancer may exhibit a unique biology.

\section{AgAINST}

\section{By Carey K. Anders}

Lineberger Comprehensive Cancer Center, University of North Carolina, Chapel Hill, North Carolina, USA

There is no question that breast cancer arising in young women is unique in many aspects. Challenges faced by young women diagnosed with breast cancer are often quite different from

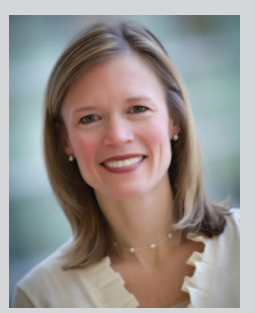

Carey K. Anders those experienced by older women. These unique challenges may include disruption of career in its early phase, child-bearing and ongoing family responsibilities, impact of therapy on sexuality and body image, and the psychosocial toll of facing a lifethreatening illness at a young age. Historically, multiple studies have shown that younger women tend to experience worse breast cancer outcomes as compared to their older counterparts [1-3]; however, the reason for this observation is not entirely understood.

Immunohistochemical (IHC) and gene expression profile studies have also shown that the more aggressive subtypes of breast cancer (i.e., basal-like and HER2-enriched) are overrepresented among younger women as compared with older women $[4,5]$. Analysis of 784 early-stage breast cancers, which included women aged $\leq 45$ years $(n=200)$ and women aged $\geq 65$ years ( $n=211$ ) identified distinct clinical-pathological features (low IHC estrogen receptor [ER] expression, high IHC HER-2 expression, larger tumours and higher tumour grade) in younger women [6]. Gene expression analysis indicated a significantly lower expression of ER and progersterone receptor $\mathrm{MRNA}$ and a significantly higher expression level of HER-2 and epidermal growth factor receptor (EGFR) mRNA in younger women.

A more detailed view of the biology of young women's breast tumours, obtained by analysing microarray data from several large, publicly available data sets in a non-subtype-dependent
Recommended

by

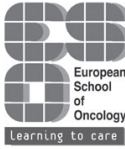

Correspondence: Marco Colleoni, M.D., Istituto Europeo di Oncologia, Via Ripamonti 435, 20141 Milan, Italy; E-Mail: marco.colleoni@ieo.it; Carey K. Anders, M.D., Assistant Professor, Hematology-Oncology, University of North Carolina at Chapel Hill, 170 Manning Dr., POB 3119, Campus Box 7305, Chapel Hill, NC 27599-7305. Telephone: E-Mail: canders@med.unc.edu. Received March 26, 2013; accepted for publication April 2, 2013. @AlphaMed Press 1083-7159/ 2013/\$20.00/0 http://dx.doi.org/10.1634/theoncologist.2013-0118 
Further evidence for a unique biology in breast cancer in young women comes from molecular studies. Young women with breast cancer have a significantly increased prevalence of the more aggressive subtypes, in particular the "basal-like" tumours $[9,10]$. Meta-analysis of prognostic signatures and gene classifiers from 20 datasets, representing over 3,500 patients aged $\leq 40$ years, indicated that distinct molecular processes including those related to immature mammary epithelial cells and growth factor signalling are overrepresented in breast cancer arising at a young age [11]. Particular genes/processes that were enriched included RANKL, c-Kit, BRCA1-mutated phenotype, mammary stem cells, luminal progenitor cells (immature mammary epithelial cell phenotype), mitogen-activated protein kinase (MAPK) and phosphoinositide 3-kinase (PI3K)-related pathways (growth factor signalling phenotype). A prognostic effect of stromal-related gene signatures was also observed, suggesting a role for the microenvironment in mediating breast cancer growth and proliferation in young women, leading to a more aggressive phenotype.

Thus, both IHC-defined subtype and molecular classification data indicate that breast cancer that develops at a young age is different biologically from that arising in older premenopausal and postmenopausal women.

\section{REFERENCES}

1. El Saghir NS, Seoud M, Khalil MK et al. Effects of young age at presentation on survival in breast cancer. BMC Cancer 2006;6:194.

2. Bharat A, Aft RL, Gao Fet al. Patient and tumor characteristics associated with increased mortality in young women ( $\leq 40$ years) with breast cancer. J Surg Oncol 2009;100:248-51.

3. Kim EK, Noh WC, Han W et al. Prognostic significance of young age ( $<$ 35years) by subtype based on ER, PR, and HER2 status in breast cancer: a nationwide registry-based study World J Surg 2011;35:1244-53.

4. Sorlie T, Tibshirani R, Parker J et al. Repeated observation of breast tumor subtypes in independent gene expression data sets. Proc Natl Acad Sci U S A 2003;100:8418-23.

5. Sorlie T, Perou CM, Tibshirani R et al. Gene expression patterns of breast carcinomas distinguish tumor subclasses with clinical implications. Proc Natl Acad Sci U S A 2001;98:10869-74.

6. Perou CM, Sorlie T, Eisen MB et al. Molecular portraits of human breast tumours. Nature 2000;406:747-52.

7. Anders CK, Hsu DS, Broadwater $\mathrm{G}$ et al. Young age at diagnosis correlates with worse prognosis and defines a subset of breast cancers with shared patterns of gene expression. J Clin Oncol 2008;26:3324-30.

8. Cancello G, Maisonneuve $P$, Rotmensz $\mathrm{N}$ et al. Prognosis and adjuvant treatment effects in selected breast cancer subtypes of very young women (<35years) with operable breast cancer. Ann Oncol 2010;21:1974-81.

9. Carey LA, Perou CM, Livasy CA et al. Race, breast cancer subtypes, and survival in the Carolina Breast Cancer Study. JAMA 2006;295: 2492-2502. 28.

10. Bauer KR, Brown M, Cress RD et al. Descriptive analysis of estrogen receptor (ER)-negative, progesterone receptor (PR)-negative, and HER2-negative invasive breast cancer, the so-called triple-negative phenotype: a populationbased study from the California cancer Registry. Cancer 2007;109:1721-172.

11. Azim HA Jr. Michiels S, Bedard PL et al. Elucidating prognosis and biology of breast cancer arising in young women using gene expression profiling. Clin Cancer Res 2012;18:1341-51. manner, indicated that breast tumours arising in younger women were enriched for 367 biologically relevant gene sets when compared with older women [6], suggesting, with the IHC data, a unique biology for breast cancer in younger women. Independent analysis of a second pooled data set, which included women aged $\leq 45$ years and women aged $\geq 65$ years confirmed the increased incidence of the more aggressive basal-like and HER-2 enriched subtypes in younger women [7]. However, when correcting for significant clinical-pathological and histopathological features including grade, nodal status, ER status and intrinsic breast cancer subtype, adjusted models yielded negligible gene differences between breast tumours arising from defined age groups of $\leq 45$ versus $\geq 65$ years $[6,7]$. As is standard in the field, this finding was replicated in an independent data set as part of this analysis, further confirming these results.

Based on these results, age alone does not appear to offer an additional layer of biological complexity above that of breast cancer subtype and grade. These data support the argument that the biology of young women's breast tumours may not be unique, but rather an overrepresentation of aggressive, biologically driven subtypes is accounting for the disparities observed in outcome by age.

While the information generated by gene expression profiling is compelling, many unanswered questions remain, including: (1) why are younger women more prone to aggressive subtypes of breast cancer; (2) what is the role of the microenvironment; (3) how does breast density and/or other factors (i.e., breastfeeding, parity) contribute to these findings; and (4) will disparities in outcome persist in the era of modern targeted therapies-all areas deserving of further research.

\section{REFERENCES}

1. Adami HO, Malker B, Holmberg $L$ et al. The relation between survival and age at diagnosis in breast cancer. N Engl J Med 1986;315:559-63.

2. Chung M, Chang HR, Bland KI et al. Younger women with breast carcinoma have a poorer prognosis than older women. Cancer 1996;77:97-103.

3. Bharat A, Aft RL, Gao F et al. Patient and tumor characteristics associated with increased mortality in young women ( $\leq 40$ years) with breast cancer. J Surg Oncol 2009;100:248-51.

4. Bauer KR, Brown M, Cress RD et al. Descriptive analysis of estrogen receptor (ER)-negative, progesterone receptor (PR)-negative, and HER2-negative invasive breast cancer, the so- called triple-negative phenotype: a population-based study from the California cancer Registry. Cancer 2007;109:1721-8.

5. Cancello G, Maisonneuve P, Rotmensz $\mathrm{N}$ et al. Prognosis and adjuvant treatment effects in selected breast cancer subtypes of very young women (<35years) with operable breast cancer. Ann Oncol 2010;21:1974-81.

6. Anders CK, Hsu DS, Broadwater G et al. Young age at diagnosis correlates with worse prognosis and defines a subset of breast cancers with shared patterns of gene expression. J Clin Oncol 2008;26:3324-30.

7. Anders $\mathrm{CK}$, Fan C, Parker JS et al. Breast carcinomas arising at a young age: unique biology or a surrogate for aggressive intrinsic subtypes? J Clin Onco 2011;29:e18-e20. 


\section{Overall Conclusion}

The question as to whether younger patients with breast cancer exhibit a unique biology is a controversial one. All of the data presented both in favour of and against this hypothesis indicate an increased incidence of more aggressive molecular subtypes in young women with breast cancer. It may be that factors such as the cut-off age for younger patients need to be considered-perhaps a different biology underpinned by basal-like or HER-2 enriched molecular subtypes is implicated in very young patients (i.e., younger than 35 years of age). A precise consideration of the role of the stromal microenvironment may also be relevant and should be pursued. In any case, it is clear that our increased understanding of breast cancer tumour biology in younger women is starting to inform a new scientific rationale (e.g., targeting of genes like RANKL or growth factor pathways like PI3K), that may be of particular benefit to this poor-prognosis cohort of patients. 